\title{
Cento e vinte anos de totais extremos de precipitação pluvial máxima diária em Campinas, Estado de São Paulo: análises estatísticas
}

\author{
Gabriel Constantino Blain( $\left.{ }^{*}\right)$ \\ Instituto Agronômico, Centro de Pesquisa e Desenvolvimento de Ecofisiologia e Biofísica, Caixa Postal 28, 13001-970 Campinas (SP). \\ (*)Autor correspondente: gabriel@iac.sp.gov.br
}

Recebido: 28/ago./2010; Aceito: 11/abr./2011

\section{Resumo}

O objetivo do trabalho foi descrever, com base na distribuição geral dos valores extremos (GEV), a probabilidade de ocorrência associada aos totais máximos anuais de precipitação pluvial diária (Preabs), observados na localidade de Campinas entre 1890 e 2009, verificando também qual método, máxima verossimilhança ou momentos-L, resultam no melhor desempenho da GEV no referido propósito. Após verificação estatística da inexistência de significativas correlações seriais, tendências e periodicidades, observou-se que a GEV pode ser utilizada na descrição das probabilidades associadas aos dados de Preabs, na localidade de Campinas. O método da máxima verossimilhança mostrou-se, por meio do teste Lilliefors e de gráficos Quantil-Quantil, o melhor algoritmo para estimação dos parâmetros da GEV. Verificou-se ganho de energia no sinal temporal da série analisada após 1990. Essa característica espectral, também verificada entre 1920 e 1935, indica concentração dos valores mais elevados de Preabs nos referidos períodos.

Palavras-chave: distribuição geral dos valores extremos, Lilliefors.

\section{Extreme daily rainfall totals observed between 1890-2009 in the weather station of Campinas, State of São Paulo, Brazil: statistical evaluations}

\section{Abstract}

The aim of the work was to evaluate the probability of occurrence of daily extreme rainfall totals during each year (Preabs), considering the time series of 1890 to 2009 in the weather station of Campinas, State of São Paulo, Brazil. The probability calculations were based on the General Extreme Value distribution (GEV) from two methods of parameter estimations: the maximum likelihood and the L-moments. After evaluating the presence of no significant serial correlation, no trends, and no periodical components within the analyzed time series, it was verified that the GEV can be used to assess the probability of occurrence of the Preabs totals in the location of Campinas. The Lilliefors test and the Quantil-Quantil plots have indicated that the maximum likelihood method can be seen as a better model as compared to the L-moments in calculating the GEV parameters. It was also observed an increasing level of the wavelet energy after the 1990s. As the period between 1920 and 1935 has shown a similar feature on the wavelet sign, it can be inferred that the higher Preabs values are concentrated during these both periods (1920-1935 and after the 1990s).

Key words: general extreme value distribution, Lilliefors. 


\section{INTRODUÇÃO}

Apesar do elevado desenvolvimento tecnológico atual, a vulnerabilidade $\left({ }^{1}\right)$ do ser humano a eventos climáticos adversos continua bastante elevada. Nesse contexto, segundo WiLKs (2006), a associação entre os totais anuais máximos de precipitação pluvial diária (Preabs) e a ocorrência de eventos como inundaçôes ou enchentes (frequentemente agravados pela ação humana), é motivação importante para estudo e modelagem probabilística dessas séries temporais meteorológicas.

Sob essa forma de análise estatística, o trabalho pioneiro de Fisher e Tippett (1928), em que podem ser encontrados os fundamentos da teoria dos valores extremos, ocupa posição de destaque. Nesse estudo, são descritos três casos particulares da distribuição generalizada de valores extremos (GEV; Jenkinson, 1955) conhecidos como Gumbel (tipo I), Fréchet (tipo II) e Weibull (tipo III) (Gumbel, 1958). Entretanto, segundo Raynal (1997), como a escolha de qual dos tipos, I, II ou III é o mais adequado aos dados sob investigação nem sempre é óbvia, a GEV sempre interessante alternativa para a investigação da estatística de valores extremos. Essa indicação de RaYnal (1997) é corroborada por NaDARAJAH e CHOI, (2007) quando afirmam que a GEV possui toda a flexibilidade contida em seus casos particulares. Com base nessa última premissa, El Adlouni et al. (2007), Pujol et al. (2007), Méndez (2007), Furió e Meneu (2010) e CanNON (2010) utilizam esse modelo estatístico em estudos probabilísticos de dados meteorológicos extremos. Sob o aspecto matemático, é interessante ressaltar que os parâmetros da referida função estatística são frequentemente estimados com base nos métodos da máxima verossimilhança (Nadarajah e Choi, 2007; Pujol et al., 2007; MÉndez, 2007; SANsigolo, 2008) ou dos momentos-L (Hosking e Wallis, 1997; Queiroz e Chaudhry, 2006; WiLKs, 2006).

Para SANSigolo (2008), a teoria dos valores extremos assume, conceitualmente, a inexistência de variaçóes sistemáticas, como tendências e periodicidades, na série sob análise. Nesse aspecto, Wirks (2006) indica que um problema relacionado à aplicação dessa teoria é que nem sempre os dados constituintes de uma série de valores extremos são oriundos do mesmo processo físico. Contudo, WiLKs (2006) também ressalta que, empiricamente, o uso da GEV é frequentemente adequado, mesmo quando nem todas as exigências conceituais são satisfeitas. Conforme SANSIGolo (2008), distribuições paramétricas são simplesmente funçóes analíticas utilizadas no estudo da variabilidade de determinadas variáveis. Sob esse último aspecto, a verificação da possibilidade de utilização da GEV na caracterização probabilística de eventos extremos de precipitaçáo pluvial em uma das séries meteorológicas mais antigas do país (Campinas, Estado de São Paulo, 1890 a 2009) torna-se extremamente importante.

Dessa forma, considerando a relevante contribuiçáo que a análise estatística dessa série do IAC pode fornecer diante de uma das maiores vulnerabilidades da sociedade, o objetivo do trabalho foi descrever, com base na distribuiçáo geral dos valores extremos, a probabilidade de ocorrência associada aos dados de Preabs na localidade de Campinas entre 1890 e 2009. O desempenho dessa função probabilística, quando calculada com base nos métodos da máxima verossimilhança e/ou dos momentos-L, também foi avaliado.

\section{MATERIAL E MÉTODOS}

Foram utilizados dados anuais de precipitação pluvial absoluta pertencentes ao Instituto Agronômico (Figura 1; IAC/SAA-SP; Campinas 1890 a 2009; 22054'S; 4705'W; 669m). Conforme Vicente e Nunes (2004), este município localiza-se próximo ao Tropico de Capricórnio com influência tanto de sistemas tropicais quanto extratropicais. Para Monteiro (1973), o sistema polar atlântico comanda o ritmo climático da regiâo em que a localidade de Campinas está inserida.

A função densidade de probabilidade utilizada para descrever analiticamente a série de Preabs foi denominada distribuição geral de valores extremos descrita por:

$$
\begin{aligned}
& f(\text { Preabs })=\frac{1}{\beta}\left[1+\frac{k(\text { Preabs }-\zeta)^{1-\frac{1}{k}}}{\beta}\right] \exp \left\{\left[1+\frac{k(\text { Preabs }-\zeta)^{1}}{\beta}\right]^{-\frac{1}{k}}\right\}(1) \\
& \text { Para }
\end{aligned}
$$

$1+\frac{\mathrm{k}(\text { Preabs })-\zeta)}{\beta}>0$

em que:

$\zeta$ é o parâmetro de localização;

$\beta$ é o parâmetro de escala e;

$\mathrm{k}$ é o parâmetro de forma.

A equação 1 pode ser integrada analiticamente fornecendo a função cumulativa de probabilidade:

$$
F(\text { Preabs })=\exp \left\{-\left[1+\frac{k(\text { Preabs }-\zeta)}{\beta}\right]^{-1 / k}\right\}
$$

${ }^{(1)}$ Grau que um sistema é suscetível, ou incapaz de enfrentar efeitos adversos. É uma função entre a natureza, a magnitude e o percentual de uma variação que o sistema é exposto, sua sensitividade, sua capacidade adaptativa. Depende, ainda, de uma relação complexa de situaçôes econômicas, sociais, culturais e políticas, que são configuradas por eventos extremos já enfrentados por determinadas sociedades (IPCC, 2001) 
Os parâmetros da equação 1 foram estimados pelos métodos da máxima verossimilhança e dos momentos-L.

A função inversa da GEV pode ser escrita a fim de indicar o valor Preabs correspondente a um dado quantil q:

$\mathrm{F}^{-1}(\mathrm{q})=\xi+\frac{\beta}{\mathrm{k}}\left\{[-1 \mathrm{n}(\mathrm{q})]^{-\mathrm{k}}-1\right\}$

Um aspecto importante a ser considerado é o fato de que a presença de persistência temporal, de tendências temporais e de periodicidades em uma amostra, interfere na estrutura de probabilidade associada aos dados. Com isso foram inicialmente realizadas investigaçóes sobre a presença dessas componentes na série de Preabs por meio dos testes Run, conforme descrito em SANSIgOlo e Kayano (2010), e Mann-Kendall (Mann, 1945; Kendall e Stuart, 1967; Subash et al., 2010; Blain, 2010). A significância associada aos resultados desses dois métodos foi descrita por meio do valor p. A periodicidade dos picos de variância da série foi investigada com base na análise de ondaleta, conforme descrita em ToRrence e Cомpo (1998) e utilizada por Reboita et al. (2006) e BLAIN (2009). Essa análise foi feita utilizando o algoritmo elaborado por C. Torrence disponível em http://paos.colorado.edu/research/wavelets, na linguagem utilizada pelo software Matlab. A determinação da significância estatística dos picos espectrais de variância foi realizada conforme Torrence e Compo (1998).

O teste de aderência Kolmogorov-Smirnov foi utilizado para verificar o grau de ajuste das distribuiçóes empíricas à GEV. Conforme indicado por Wilks (2006), esse método compara a curva de probabilidade acumulada empírica à curva teórica evitando a divisão delas em classes discretas, conforme equação 4 .

$\operatorname{Dmax}=M a x=\mid F^{\prime}($ Preabs $)-F($ Preabs $) \mid$

em que:

F'(Preabs) é a frequência acumulada empírica dos valores de Preabs;

F(Preabs) é dado pela equação 2 .

Conforme ressaltado por Crutcher (1975), Wilks (2006), Steinskog et al. (2007) e Vlcek e Huth (2009), o método original do Kolmogorov-Smirnov não pode ser aplicado quando o período utilizado para a realizaçáo deste teste é o mesmo adotado para estimação dos parâmetros do modelo teórico sob investigação. Para VLCEK e Huth (2009) essa última situação, frequentemente enfrentada em aplicaçóes práticas, eleva a probabilidade de ocorrência do erro estatístico tipo II. Nesse caso, esse teste de aderência deve ser modificado conforme LiLLIEFors (1967; 1969). Sob essa última condição esse método é usualmente denominado Lilliefors. Segundo Crutcher (1975) e WiLKs (2006), o valor crítico relativo à rejeição/aceitação da hipótese de que as probabilidades de ocorrência dos valores de Preabs podem ser estimadas com base na GEV $\left(\mathrm{H}_{0}\right.$ associada ao teste Lilliefors), dependerá: a) do nível de significância adotado (para o estudo p $>0,05$ resultará na não rejeição de $\mathrm{H}_{0}$ ); b) do número de dados amostrais; c) dos valores dos parâmetros estimados. Segundo Wilks (2006), os limites críticos do teste Lilliefors são usualmente determinados utilizando-se simulaçôes estatísticas. Neste estudo, esse procedimento adotou a geração de Ns=10000 amostras sintéticas oriundas das GEV (com parâmetros conhecidos e com base no método geração por inversão de números aleatórios distribuídos de forma não uniforme). Para cada uma das 10000 amostras foram entáo estimados novos parâmetros dessa distribuição. $\mathrm{O}$ ajuste entre as curvas de probabilidade associadas a essas amostras e a $\mathrm{F}$ (Preabs) foi avaliado utilizando-se a equação 4. Essa última etapa resultou na elaboração de uma curva com 10000 valores. Uma vez que a $\mathrm{H}_{0}$ é, para as simulaçóes estatísticas, verdadeira por definição (WILKs, 2006), o valor crítico associado ao teste Lilliefors, para dada significância p, é o quantil 1- p dessa curva gerada pela expressão 4 (95\% ou 9500 séries sintéticas, no presente caso). Descrições mais detalhadas sobre esse método são dadas em Wilks (2006).

Contudo, conforme SANsilogo (2008), o teste Lilliefors é somente adequado para verificar a parte central das distribuiçôes. Com isso os gráficos Quantil-Quantil foram também utilizados para auxiliar a avaliação do desempenho da GEV sob o presente objetivo. Embora WILKS (2006) indique que tais gráficos sejam conceituados como métodos qualitativos de verificação do uso de modelos paramétricos para estimação das probabilidades associadas a amostras de dados, a análise de regressão linear simples e o índice (d) de concordância de Willmott (Willmott, 1985) foram utilizados para auxiliar sua interpretação. O teste Lilliefors e os gráficos Quantil-Quantil também foram empregados para determinar qual dos métodos, máxima verossimilhança e momentos-L, resultam na GEV com melhor desempenho. Após essa determinação, o período de retorno [R(Preabs)] associado aos valores de Preabs pôde ser estimado por:

$\mathrm{R}($ Preabs $)=\frac{1}{\mathrm{w}(1-\mathrm{F}(\text { Preabs }))}$ para $\mathrm{w}=\mathrm{ano}^{-1}$

\section{RESULTADOS E DISCUSSÃO}

\section{Persistência temporal, periodicidades e tendências climáticas.}

A aplicação do teste Run indicou que a série de Preabs pode ser considerada livre de correlaçáo serial ou persistência temporal, uma vez que o valor final de sua estatística $(1,02)$, associada ao valor $\mathrm{p}$ de significância 0,30 , 
encontra-se consideravelmente distante dos limites críticos usual e arbitrariamente adotados na literatura ( $\mathrm{p} \leq$ $0,05$ ou $\mathrm{p} \leq 0,10)$ como significativos. A estatística de Mann-Kendall ao apresentar o valor de 0,71, associado a $\mathrm{p}=0,48$, também não indica a presença de significativas tendências climáticas de elevação nos totais de Preabs observados entre 1890 e 2009.

A análise do espectro local da ondaleta e, em especial, da potência global da ondaleta (Figura 2a,b) indica inexistência de significativas periodicidades nos picos de variância do sinal temporal da Preabs. Essa última característica, associada aos resultados dos testes Run e MannKendall, permite inferir que o uso da GEV para estimação

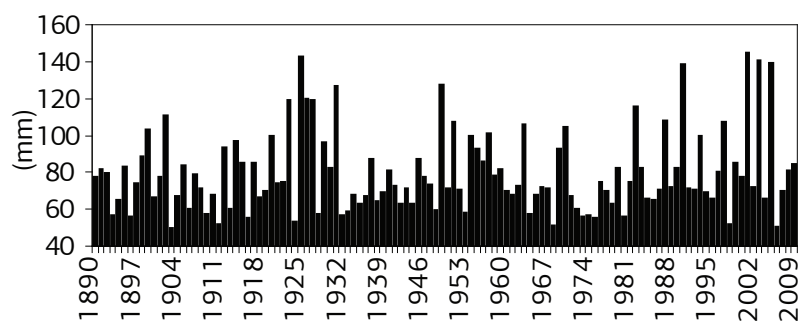

Ano

Figura 1. Dados anuais do maior valor diário de precipitação pluvial - Campinas, Estado de Sáo Paulo (IAC/APTA/SAA; 1890 a 2009). da probabilidade de ocorrência dos valores de Preabs, uma vez comprovado o ajuste aos dados empíricos, pode ser vista como importante etapa no entendimento da variabilidade climática da localidade de Campinas, auxiliando o gerenciamento de atividades relacionadas a essa variável meteorológica.

Nas escalas 2-8 e 10-20 anos (Figura 2c) observa-se elevação na energia da ondaleta nos últimos 20 anos da série sob análise (após 1990). Nota-se, também, que esse ganho de potência ocorre após um período de relativa baixa energia no sinal temporal (1935 a 1990; escalas 2-8 e 10-20 anos), fazendo com que o sinal do espectro da ondaleta após 1990 seja semelhante ao observado entre 1920 e 1935 . Em outras palavras, e do ponto de vista de inferências estatísticas, existe indicação de que a variabilidade da Preabs entre 1990 e 2009 esteja ocorrendo de forma similar à observada nos anos de 1920 a 1935 . Futuros estudos poderão verificar as possíveis forçantes físicas associadas a essa última característica.

\section{Ajuste à distribuição geral dos valores extremos}

$\mathrm{O}$ resultado do teste Lilliefors indica que a GEV pode ser utilizada na descrição analítica das probabilidades associadas (a) Espectro de energia local

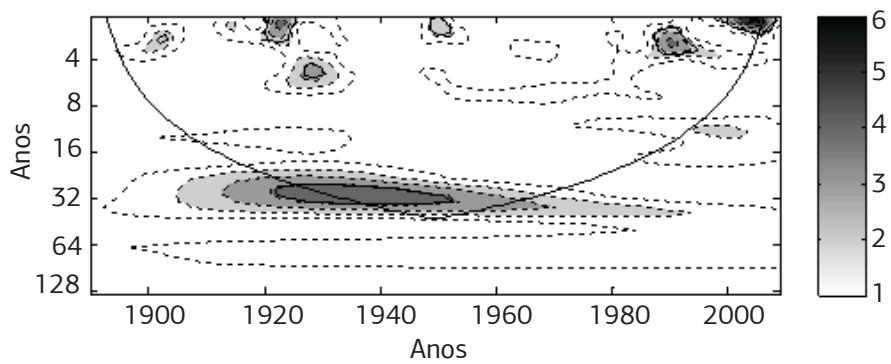

(c) Variância Média (b) Potência global

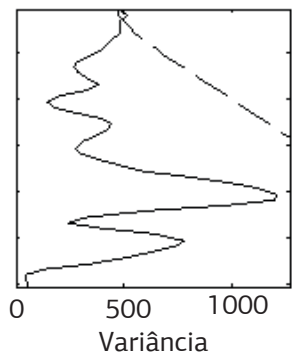

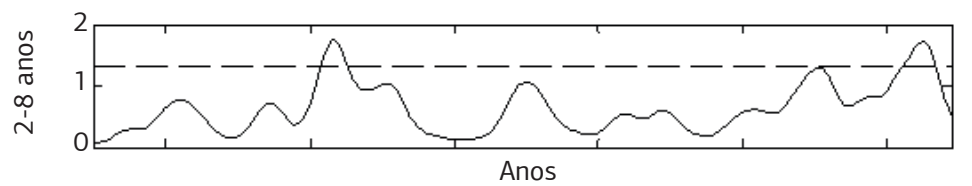

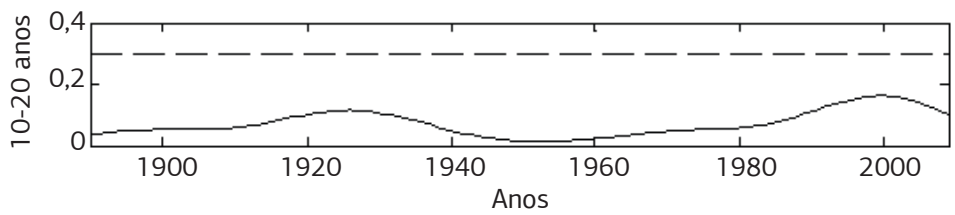

Figura 2. (a) Espectro de energia local da ondaleta dos dados diários extremos da série de precipitaçáo anual da localidade de Campinas (SP). Contornos tracejados correspondem a valores de variância normalizados $\left(\mathrm{mm}^{2}\right)$ variando de $1 \mathrm{a} 10$, conforme representado na barra horizontal. Contornos sombreados englobam áreas com variâncias significativas ao nível de $95 \%$ de confiança. A curva em forma de U representa o cone de influência, sob a qual o efeito de borda é importante; (b) Espectro ou potência global de ondaleta; c) Variância média. 
à Preabs na localidade de Campinas. Tanto o método da máxima verossimilhança quanto o momentos-L resultam em bom desempenho da GEV na estimação da probabilidade de ocorrência associada aos dados de Preabs. O valor final da equação 4 foi 0,1026 e 0,042 para os métodos momentos-L e máxima verossimilhança, respectivamente. Essas estatísticas são bastante inferiores ao limite crítico de rejeição de $\mathrm{H}_{0}$ a 5\% (Figura 3). Verifica-se, também, que o menor valor da equação 4 , relativo à adoção do método da máxima verossimilhança, indica que o mesmo proporcionou a curva de probabilidade acumulada paramétrica mais próxima à curva de probabilidade acumulada empírica. Com base nos momentos-L, os valores dos parâmetros de forma, escala e localização foram 0,1301, 14,6645, e 68,6628, respectivamente. Com base no método da máxima verossimilhança esses valores foram de 0,1640, 14,2121 e 68,5021, respectivamente.

Corroborando a análise baseada nos valores da equação 4, os gráficos Quantil-Quantil (Figura 4) apontam melhor desempenho do método da máxima verossimi-
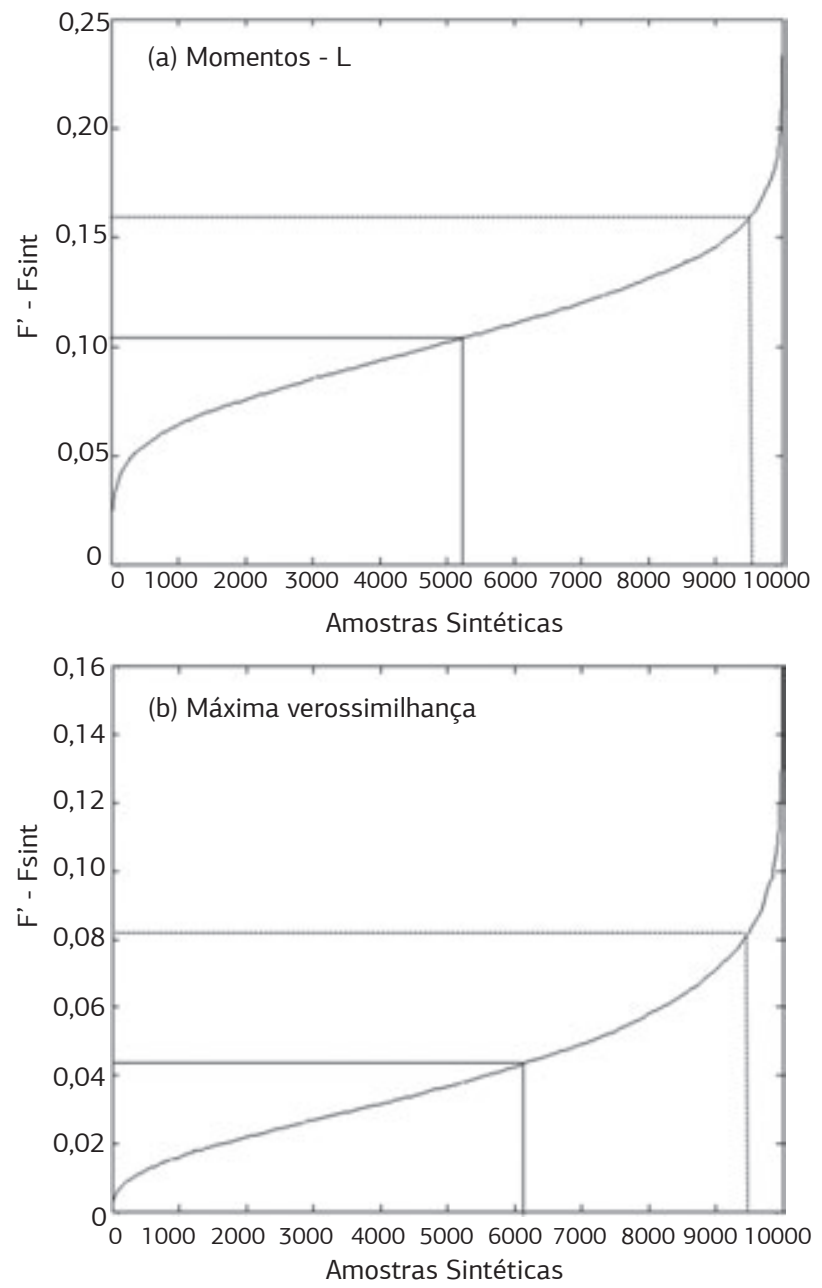

Figura 3. Teste Lilliefors aplicado a dados diários extremos da série de precipitação anual da localidade de Campinas (SP), considerando a ajuste a distribuição geral dos valores extremos com base em dois métodos de estimação dos parâmetros. As linhas sólidas representam o valor final do teste. As linhas pontilhadas representam o valor crítico a 5\% de significância.

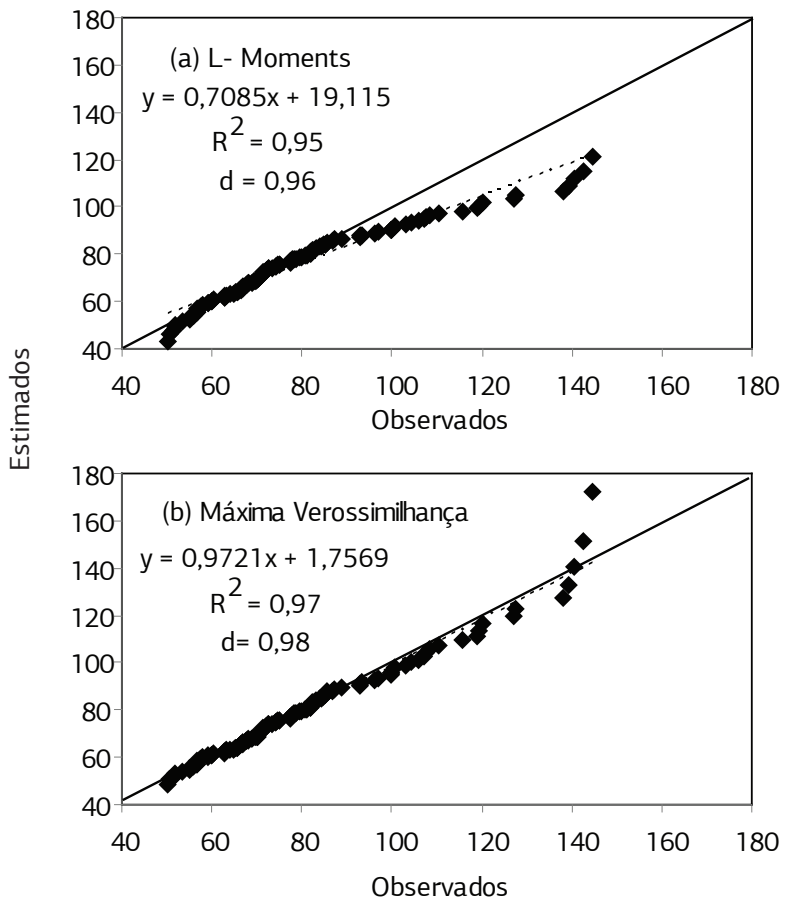

Figura 4. Gráficos Quantil-Quantil relativos ao ajuste dos 120 dados diários extremos da série de precipitação anual da localidade de Campinas (SP), a distribuição geral dos valores extremos com base em dois métodos de estimação dos parâmetros.

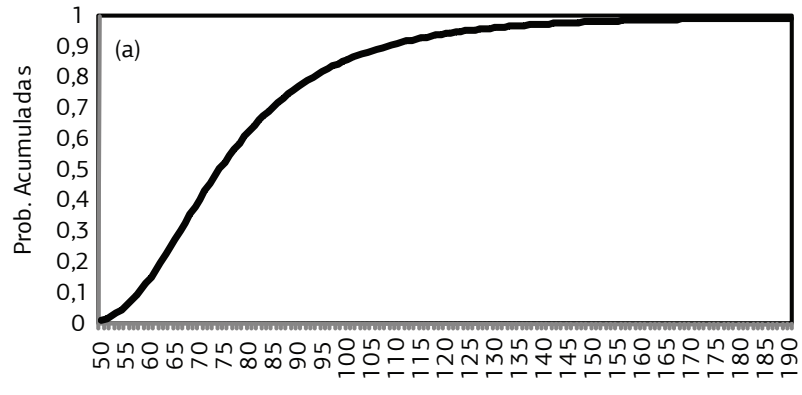

Precipitação absoluta diá ria $(\mathrm{mm})$

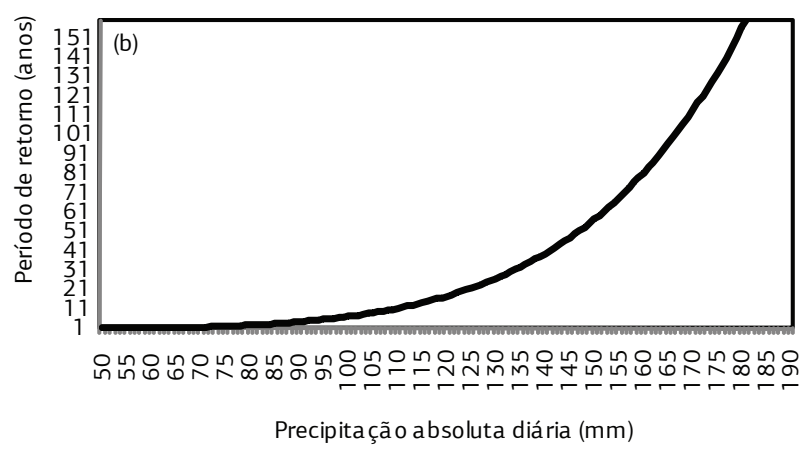

Figura 5. (a) Curva de Probabilidade acumulada de totais diários extremos de precipitação pluvial [F(Preabs)], estimada por meio da distribuição geral dos valores extremos, para a série de valores anuais absolutos do Instituto Agronômico de Campinas (1890-2009); (b) período de retorno [R(Preabs)], em anos, associado a probabilidade acumulada de cada evento descrito em a. $[\mathrm{R}($ Preabs $)=1 /(1-$ $\mathrm{F}$ (Preabs)]. 
lhança. Sob esse aspecto, verifica-se que o coeficiente angular e o intercepto, obtidos após a análise de regressão linear simples, permanecem mais próximos à bissetriz do ângulo formado pelos eixos $\mathrm{x}$ e $\mathrm{y}$ na figura $4 \mathrm{~b}$ do que na figura 4a. Os valores do índice d de Willmott e do coeficiente resultante da regressão linear $\left(\mathrm{r}^{2}\right)$ também indicam melhor desempenho do referido método. Nesse aspecto, é interessante ressaltar SANSIGOLO (2008) que, investigando a probabilidade de ocorrência de valores extremos de precipitação pluvial, entre outros elementos meteorológicos, na localidade de Piracicaba, afirma que o método da máxima verossimilhança é o melhor algoritmo de estimação dos parâmetros de distribuições teóricas.

A elevada potencialidade de ocorrência de episódios extremos de precipitação pluvial diária, na localidade de Campinas, torna-se evidente após quantificação das probabilidades e dos períodos de retorno associados aos dados de Preabs. O valor $100 \mathrm{~mm}$ de Preabs, conceituado por Vicente e Nunes (2004) como evento extremo, é associado a um período de retorno próximo a (apenas) sete anos (Figura 5). Em adição, VInCENT e Nunes (2004) também afirmam que o crescimento urbano desordenado do município de Campinas contribui para elevação da frequência e da intensidade de eventos como enchentes (entre outros). Dessa forma, o conhecimento das probabilidades de ocorrência associadas aos dados de Preabs, bem como a análise de sua variabilidade ao longo dos 120 anos utilizados, constitui-se em relevantes contribuiçôes junto ao gerenciamento de enchentes e inundaçóes na região de Campinas. É também oportuno ressaltar que as Equaçōes 3 e 5 podem ser utilizadas para estimar o total de Preabs associado a determinado período de retorno. Como exemplo, escolhendo arbitrariamente os valores 200, 500 e 1000 anos (período de retorno), obtém-se, respectivamente, os seguintes totais diários de Preabs: 188, 222 e $250 \mathrm{~mm}$.

\section{CONCLUSÃO}

A série temporal constituída dos totais máximos de precipitação pluvial diária registrados a cada ano na localidade de Campinas, entre 1890 e 2009, não representa componentes significativas de correlação serial, tendências temporais ou periodicidades. Esses dados podem ter sua probabilidade de ocorrência descrita pela distribuição geral dos valores extremos em que o método da máxima verossimilhança é indicado como a melhor opção, quando comparado ao método dos momentos-L, para estimação dos parâmetros dessa distribuição teórica na referida série temporal.

Observa-se ganho de energia no sinal temporal da série analisada após 1990. Essa característica espectral, também verificada entre 1920 e 1935, indica concentração dos valores mais elevados da série de Preab nos referidos períodos.

\section{AGRADECIMENTO}

O autor cordialmente agradece a Dr. ${ }^{a}$ Regina Célia de Matos Pires pela valiosa contribuição para a realizaçáo deste estudo.

\section{REFERÊNCIAS}

BLAIN, G.C. Considerações estatísticas relativas à oito séries de precipitaçáo pluvial da Secretaria de Agricultura e Abastecimento do Estado de São Paulo. Revista Brasileira de Meteorologia, v.24, p.12-23, 2009.

BLAIN, G.C. Séries anuais de temperatura máxima média do ar no Estado de São Paulo: variaçôes e tendências climáticas. Revista Brasileira de Meteorologia, v.25, p.114-124, 2010

CANNON, A.J. A flexible nonlinear modeling framework for nonstationary generalized extreme value analysis in hydroclimatology. Hydrological Process, v.24, p.673-685, 2010

CRUTCHER, H.L. A note on the possible misuse of the Kolmogorov-Smirnov test. Journal of Applied Meteorology, v.14, p.1600-1603, 1975.

EL ADLOUNI, S., OUARDA, T.B.M.J., ZHANG, X., ROY, R., BOBÉE, B. Generalized maximum likelihood estimators for the nonstationary generalized extreme value model. Water Resources Research, v. 43, p.1-13, 2007.

FISCHER, R.A.; TIPPETT, L.H.C. Limiting forms of the frequency distribution of the largest or smallest member of a sample. Proceedings of the Cambridge Philosophical Society, v.14, p.180-190, 1928.

FURIÓ, D.; MENEU, V. Analysis of extreme temperatures for four sites across Peninsular Spain. Theoretical Applied Climatology, v.24, p. $1-17,2010$

GUMBEL, E.J. Statistics of Extremes. New York: Columbia University Press, 1958. 375p.

HOSKING, J.R.M.; WALLIS, J.R. Regional Frequency Analysis, Cambridge: Cambridge University Press, 1997. 224p.

JENKINSON, A.F. The frequency distribution of the annual maximum (or minimum) values of meteorological elements. Quarterly Journal of the Royal Meteorological Society, v.81, p.159$171,1955$.

IPCC. Climate Change 2001: Impacts, Adaptation and Vulnerability. Cambridge: Cambridge University Press, 2001, 1032 p.

KENDALL, M.A.; STUART, A. The advanced theory of statistics. 2.ed. Londres: Charles Griffin, 1967. v.2, 690p. 
LILLIEFORS, H.W. On the Kolmogorov-Smirnov test for normality with mean and variance unknown. Journal of the American Statistical Association, v.62, p.399-402, 1967.

LILLIEFORS, H.W. On the Kolmogorov-Smirnov test on the exponential distribution with mean unknown. Journal of the American Statistical Association, v.64, p.387-389, 1969.

MANN, H.B., Non-parametric tests against trend. Econometrica, v.13, p.245-259, 1945.

MÉNDEZ, F.J.; MENÉNDEZ, M.; LUCEÑO, A.; LOSADA, I.J. Analyzing Monthly Extreme Sea Levels with a Time-Dependent GEV Model. Journal of Atmospheric and Oceanic Technology. v.24, p.894-911, 2007

MONTEIRO, C.A.F. A dinâmica climática e as chuvas no estado de São Paulo: estudo geográfico sob forma de Atlas. São Paulo: IGEOG-USP, 1973. 154p.

NADARAJAH, S., CHOI, D., Maximum daily rainnfall in South Korea. Journal of Earth System Science, v.116, p.311-320, 2007.

PUJOL, N.; NEPPEL, L., SABATIER, R. Regional tests for trend detection in maximum precipitation series in the French Mediterranean region Hydrological Sciences Journal, v.52, p.952973, 2007.

QUEIROZ, M.M.F.; CHAUDHRY, F.H. Análise de eventos hidrológicos extremos, usando-se a distribuição GEV e momentos LH. Revista Brasileira de Engenharia Agrícola e Ambiental, v.10, p.381-389, 2006.

RAYNAL, J.A., Sobre el uso del dominio de atracción para la identificación de valores extremos para máximos. Ingenieria Hidrólica en Mexico, v.12, p.57-62, 1997.

REBOITA, M.S.; KRUSCHE, N.; PICCOLI, H.C. Climate variability in Rio Grande, RS, Brazil: A quantitative analysis of contributions due to atmospheric systems. Revista Brasileira de Meteorologia, v.21, p.256-270, 2006.

SANSIGOLO, C.A. Distribuiçóes de extremos de precipitação diária, temperatura máxima e mínima e velocidade do vento em Piracicaba, SP (1917-2006). Revista Brasileira de Meteorologia, v.23, p.341-346, 2008.

SANSIGOLO, C.A.; KAYANO, M.T. Trends of seasonal maximum and minimum temperatures and precipitation in Southern Brazil for the 1913-2006 period. Theoretical and Applied Climatology, v.101, p.209-216, 2010.

SUBASH, N.; SIKKA, A.K.; ram MAHAN, H.S. An investigation into observational characteristics of rainfall and temperature in central northeast India: a historical perspective 1889-2008. Theoretical and Applied Climatology, v.103, p.305-319, 2010.

STEINSKOG, D.J., TJØSTHEIM, D.B., KVAMSTØ, N.G. A cautionary note on the use of the Kolmogorov-Smirnov test for normality. Monthly Weather Review, v.135, p. 1151-1157, 2007.

TORRENCE, C.; COMPO, G.P. A Practical Guide to Wavelet Analysis. Bulletins of American Meteorological Society, v.79, p.6178, 1998.

VICENTE, A.K., NUNES, L.H. Extreme precipitation events in Campinas, Brazil. Terrae, v.1, p.60-62, 2004.

VLČEK, O.; HUTH R. Is daily precipitation Gamma-distributed? Adverse effects of an incorrect application of the KolmogorovSmirnov test. Atmospheric Research, v.93, p. 759-766, 2009.

WILKS, D.S. Statistical methods in the atmospheric sciences. 2.ed. San Diego: Academic Press, 2006. 629p.

WILLMOTT, C.J.; ACKLESON, S.G.; DAVIS, J.J.; FEDDEMA, K.M.; KLINK, D.R. Statistics for the evaluation and comparison of models. Journal of Geophysical Research, v.90, p.8995-9005, 1985. 